\title{
Open Reduction And Internal Fixation Of Ankle Fractures - Is Timing Of Surgery Important?
}

\author{
Faaiz Ali Shah, Mian Amjad Ali, Sajid Akhtar, Abbas Ali
}

\begin{abstract}
- - - - - - - - - - - - - - - - - - - - - - - - - - -
ABSTRACT:

Objective: To determine the frequency of surgical site infection in ankle fractures fixed earlier or delayed.

Methodology: This retrospective cohort study was conducted in the department of Orthopaedics and Traumatology Lady Reading Hospital Peshawar from March 2016 to August 2018. The medical records of all the patients with ankle fractures fulfilling the inclusion criteria were analyzed retrospectively. Demographic details of the included subjects, time of surgery and frequency of surgical site infection was noted.
\end{abstract}

Results: A total of 128 patients with mean age 38.8 years \pm SD 9.76(range 18-58 years) were included in our study. Male patients were $99(77.3 \%)$ while female were $29(22.6 \%)$. The number of patients who had open reduction and internal fixation (ORIF) within the initial 24 hours after sustaining the fractures were $12(9.3 \%)$ in number,58(45.6\%) patients had surgery in 24 to 48 hours, $40(31.4 \%)$ in 3 to 7 days and 18 (14.1\%) had surgery in 8 to 14 days after the injury.The frequency of surgical site infection(SSI) was $42.5 \%$ (17/40) in patients operated in 3 to 7 days, $44.4 \%(8 / 18)$ in patients operated in 8 to 14 days while no surgical site infection was reported in 54.6\% (70/128) patients operated in 24 to 48 hours. $(P$ value $<0.05)$.

Conclusion: The timing of ankle fracture surgery is very important. Open reduction and internal fixation of ankle fractures done earlier had no surgical site infection. Delayed fixation is associated with higher frequency of surgical site infection.

Key Words: Ankle fracture, open reduction and internal fixation(ORIF), surgical site infection(SSI)

\section{INTRODUCTION:}

Ankle fractures accounts for about $10 \%$ of all the fractures ${ }^{1}$. Open reduction and internal fixation(ORIF) of ankle fractures give better results than non operative treatment ${ }^{2,3}$. The most common complication of ankle fracture surgery is surgical site infection(SSI) which inhibits fracture union and adversely affect the functional outcome of the ankle joint ${ }^{4,5}$. Patients with surgical site infection have prolonged hospital stay and frequent re admission rates ${ }^{6}$. The reported frequency of surgical site infection after open reduction and internal fixation of ankle fracture in literature is $1.4 \%$ to $13.0 \%$ with superficial infection accounting for $3.0 \%$ to $10.0 \%$ and deep infection $1.0 \%$ to $6.8 \%{ }^{7-9}$. Various risk factors like older age, obesity, smoking, diabetes and increase operating time have been identified to be associated with increase surgical site infection after ankle fracture surgery ${ }^{7,10}$ but the

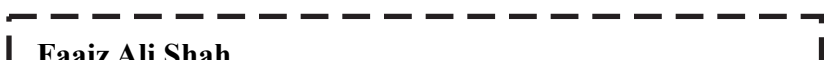

I Assistant Professor Orthopaedics

Lady Reading Hospital Peshawar

I Email: faaizalishah@yahoo.com

I Mian Amjad Ali

I Professor \& Head Department Of Orthopaedics

I Lady Reading Hospital Peshawar

I Sajid Akhtar

I Senior Registrar Orthopaedics

Saidu Medical College Swat

Abbas Ali

District Orthopaedic Surgeon

I District Headquarter Hospital Charsada

I

Received: 27-08-18

Accepted: 07-12-18 effects of delay in surgical timing on the frequency of surgical site infection is not yet clear ${ }^{11}$. Some studies ${ }^{12,13}$ reported no relationship between timing of surgery and surgical site infection while others ${ }^{11,14}$ documented an increase frequency of surgical site infection in patients who were operated late. The surgical outcome of ankle fractures have been addressed by many studies in our set up but unfortunately their main focus was either on functional outcome, length of hospital stay or cost and no study could evaluate the effects of delayed surgery on the frequency of surgical site infection. The objective of this study was, therefore to retrospectively document the frequency of surgical site infection in ankle fractures fixed earlier or delayed. Our hypothesis was that the frequency of surgical site infection would be lower in fractures if fixed earlier than delayed. The results of our study will help us not only in preoperative counseling of the patient or his family but also help us in formulating standard guidelines for managing ankle fractures in our set up.

\section{METHODOLOGY:}

Our study was a retrospective Cohort study conducted in the Department of Orthopaedics and Traumatology Lady Reading Hospital Peshawar. The research protocols were approved by the Ethical Review Board of the hospital before the commencement of our study. The medical records of all the patients who had ankle fracture surgery (open reduction and internal fixation) in our department from March 2016 to August 2018 was retrospectively analyzed. Adult patients (18 years and above) of closed ankle fractures with complete medical records and radiographs were included in the study. 
Patients with calcaneus, talus or tarsal bone fractures, pathological ankle fractures, other associated fractures requiring surgical intervention and ankle fractures of more than two weeks old were excluded from the study. The demographic variables of the included subjects like age, gender, side of injury, mechanism of injury, fracture type, time duration between the injury and surgery were noted. Pre-operative $\mathrm{x}$ rays were examined and fractures were classified according to Weber classification for ankle fractures ${ }^{15}$. The record showd that a uniform standard surgical protocol of open reduction and internal fixation(ORIF) was adopted for all the patients. All the surgeries were performed by the same surgical team with an experience of more than five years in foot and ankle surgery. General or spinal anaesthesia was used for surgery. Pneumatic tourniquet was used in all cases. Preoperative antibiotis (Inj Ceforuxime $1.5 \mathrm{gm}$ ) was given to all the patients. The operation notes indicated that depending upon fracture type, an adequate skin incision was used to fix the fibula first with appropriate length of $1 / 3$ tubular plate while malleolar screws and Kwires were used to fix medial malleolus. Syndosmotic screw was used in cases of syndosmotic disruption. Posterior malleolar fracture was fixed with cancellous screw when indicated. The wound was closed in two layers with vicryl and polypropylene suture material. Post-operative $\mathrm{x}$-rays were seen to confirm types of implant and adequacy of fracture reduction. All the patients were discharge home on the second or third post-operative day with intravenous antibiotics (Ceforuxime 1.5 gm twice daily) for three days and backslab to the foot and leg. At two weeks follow up stitches were removed and back slab discontinued. From the follow up records details of onset of surgical site infection, antibiotic use and wound dressing, culture and sensitivity reports, readmission and resurgery rates were recorded. Surgical site infection(SSI) was classified according to the Center for Disease Control(CDC) guidelines ${ }^{16}$. The medical records of the infected patients were checked in detail and infection of the skin and subcutaneous tissues indicated by inflammation of the incision site was classified as superficial surgical site infection while infection of the muscles and fascia with discharge, abscess or dehiscence was classified deep surgical site infection' ${ }^{9}$. The data collected was analyzed with computer software SPSS(version 21). Continuous variables like age and time of surgery was represented as mean \pm Standard deviation. Categorical variables like gender, type of fracture and fracture side was represented as frequency and percentages. Frequency of surgical site infection in early and delayed groups were compared and $P$ value was considered significant if $<0.05$. Data presented in graph and table where necessary.

\section{RESULTS:}

The medical records of 128 patients were reviewed. The mean age of these patients were 38.8 years \pm SD 9.76(range $18-58$ years). Majority $(77.3 \%, \mathrm{n}=99)$ of our patients were male while female were 29(22.6\%).Right ankle was fractured in $74(57.8 \%)$ patients and left in 54(42.1\%). The fracture demography of our patients are shown in table 1. Open reduction and internal fixation(ORIF) of $12(9.3 \%)$ patients were done within the initial 24 hours of sustaining the fracture while $58(45.6 \%)$ patients had surgery in 24 to 48 hours, $40(31.4 \%)$ in 3 to 7 days and $18(14.1 \%$ ) had surgery in 8 to 14 days after the injury. Surgery was done under spinal anaesthesia in majority $(71 \%, \mathrm{n}=91)$ of patients while general anaesthesia was given to $37(28.9 \%)$ patients. Overall surgical site infection was noted in $25(19.5 \%)$ patients. Patients operated in 24 to 48 hours $(54.6 \%, \mathrm{n}=70)$ had no surgical site infection while $17(42.5 \%)$ patients operated in 3 to 7 days and $8(44.4 \%)$ patients operated in 8 to 14 days developed surgical site infection $(P$ value $<0.05$ ) as shown in graph 1.Superficial surgical site infection was reported in $16(64 \%)$ patients and deep infection in $9(36 \%)$ patients. All patients with superficial surgical site infection were treated with local wound care and oral antibiotics. Culture and sensitivity reports documented Staphalocoocal aurus in $7(28 \%)$ patients and mixed micro organisms in 5(20\%) patients. Re-admission and debridement under anaesthesia was noted in 7(28\%) patients with deep infection. Implants were not removed in any patient. All patients eventually healed. No in- hospital mortality was recorded.

\begin{tabular}{|c|c|c|}
\hline Aetiology & $\begin{array}{c}\text { Number of } \\
\text { patients }\end{array}$ & Percentages \\
\hline $\begin{array}{c}\text { Motor vehicle } \\
\text { accidents }\end{array}$ & 64 & $50 \%$ \\
\hline Fall & 46 & $35.9 \%$ \\
\hline Sports injury & 18 & $14 \%$ \\
\hline Weber type I & 22 & $17.1 \%$ \\
\hline Weber type II & 65 & $50.7 \%$ \\
\hline Weber type III & 41 & $32 \%$ \\
\hline
\end{tabular}

Table I: Fracture demography of our patients

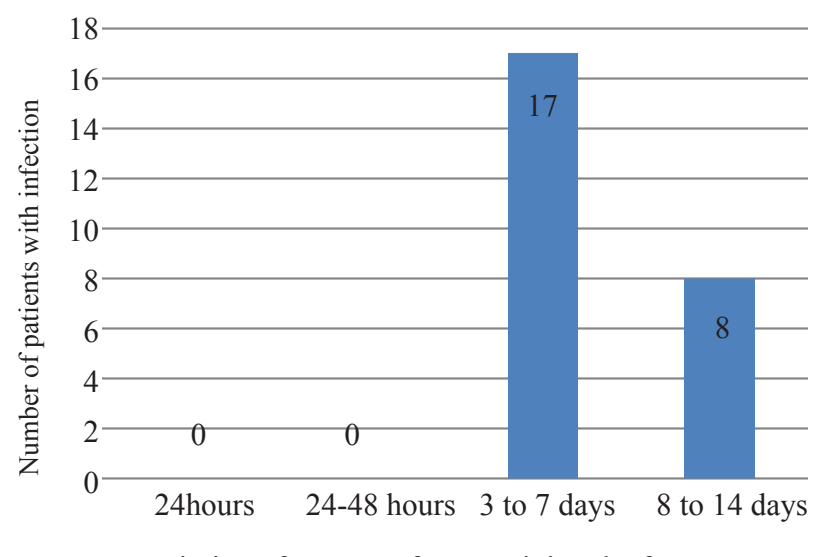

Timing of surgery after sustaining the fracture

Figure 1. Timings of surgery and the frequency of surgical site infection in patients with open reduction and internal fixation of ankle fractures. 


\section{DISCUSSION:}

The medical record of 128 patients of open reduction and internal fixation of ankle fractures indicated that no surgical site infection was noted in $54.6 \%(70 / 128)$ patients who were operated in 24 to 48 hours while $42.5 \%(17 / 40)$ patients who were operated in 3 to 7 days and $44.4 \%$ (8/18) patients operated in 8 to 14 days developed surgical site infection. Similar to our findings Schepers ${ }^{11}$ reported no infection in $60(29.2 \%)$ out of 205 ankle fractures operated within 24 hours while $16(11 \%)$ out of 145 fractures developed surgical site infection. $(\mathrm{p}=0.004)$ who were operated after 24 hours of sustaining the fractures. Saithna and Moody ${ }^{14}$ reviewed the records of 85 patients of ankle fracture surgery. They found that the frequency of wound infection in patients who had surgery after 6 days was significantly higher than those operated earlier( $3.6 \%$ vs $20.7 \%, P=0.01)$ Another study reported no infection in $24(38.7 \%)$ out of 62 patients operated within 24 hours while $6(15.7 \%)$ out of 38 patients developed infection who were fixed after 24 hours. ${ }^{17}$ Adamson 18 noted infection rate of $1.4 \%(1 / 67)$ in patients fixed within 24 hours and $9.5 \%(8 / 84)$ in patients operated after $24 \operatorname{hours}(P=0.04)$ Contrary to our observations, some researches however, reported no relationship between the timing of surgery and infection rate in ankle fracture surgery. This might be due to the differences in study designs, included subjects and evaluation of risk factors. Pietzik and Qureshi ${ }^{19}$ reported no surgical site infection in 62 patients who were operated within 48 hours while only one patient out of 21 developed infection who were operated after 48 hours. The difference however, was not statistically significant. Miller ${ }^{12}$ reported an infection rate of $4.1 \%$ after 478 ankle fracture surgery but no relationship between the timing of surgery and complication rate was found. Thangarajah and Prasad ${ }^{9}$ reported an infection rate of $24 \%$ in ankle fracture surgery, but no statistically significant association was found between timing of surgery and infection rate. $(P=0.51)$. Singh ${ }^{20}$ treated 82 patients with ankle fracture in less than 24 hours after sustaining the fractures and 132 patients more than 24 hours and noted infection rates of $7 \%$ and $11 \%(P=0.589)$ respectively. One of the largest series of ankle fracture surgery of 1011 by Naumann and Sigurdsen ${ }^{21}$ reported no statistically significant association between the wound complication rate and early or delayed surgery. They observed a safe pre-operative period of 6 days in which the patient can be optimized and surgery can be planned without an increase in frequency of surgical site infection. Sukeikh and Qaffaf ${ }^{22}$ reported infection rate of $4.2 \%(5 / 117)$ in early surgery group (24 hours) while $14.2 \%(4 / 28)$ in delayed group $(P=0.07)$ There are some limitations of our study. The design of our study was retrospective rather than randomized control trial which would have been statistically more powerful. The precise reasons for delay in surgery could not be evaluated but in our setup the usual causes for delay are late presentation of the patients to the hospital, patient initial refusal for surgery, soft tissue swelling, shortage of operation theatre and patient preparation for anaesthesia. The role of some important risk factors for surgical site infection like obesity, diabetes, operating time and smoking could not be assessed. Furthermore documentation of frequency of surgical site infection by different surgeons in the follow up period might resulted in underestimation or over estimation of surgical site infection.

\section{CONCLUSION:}

The timing of ankle fracture surgery is very important. Open reduction and internal fixation of ankle fractures done earlier had no surgical site infection. Delayed fixation is associated with higher frequency of surgical site infection. Time of surgery is a factor which can be control by the surgeon resulting in lower surgical site infection. We therefore recommend that ankle fractures should be fixed within 24 hours of sustaining the fractures.

\section{REFERENCES:}

1. Court-Brown CM, Caesar B. Epidemiology of adult fractures: A review. Injury 2006;37:691-697.

2. Vioreanu M, Dudeney S, Brophy S. Displaced ankle fractures in the geriatric population: Operative or non-operative treatment.Foot Ankle Surg 2007;13:10-4.

3. Jain S, Haughton BA, Brew C. Intramedullary fixation of distal fibular fractures: A systematic review of clinical and functional outcomes. J Orthop Traumatol 2014;15:245-54.

4. Sun Y, Wang H, Tang Y, Zaho H, Qin S, Xu L et al. Incidence and risk factors for surgical site infection after open reduction and internal fixation of ankle fracture. 2018; 97(7): 415-22.

5. Naumann MG, Sigurden U, Utvag SE, Stavem K. Functional outcome following surgical-site infections after operative fixation of closed ankle fractures. Foot Ankle Surg 2017;23(4):311-316.

6. Brennan SA, Walls RJ, Smyth E, Al Mulla T, O'Byrne JM. Tourniquets and exsanguinators: A potential source of infection in the orthopedic operating theatre? Acta Orthop. 2009;80(2):251-5.

7. Ovaska M. Complications in ankle fracture surgery. Acta Orthop Suppl 2015;86(358):1-3.

8. Zalavras C G, Christensen T, Rigopoulos N, Holtom P, Patzakis $\mathrm{M} J$. Infection following operative treatment of ankle fractures.Clin Orthop Relat Res. 2009;467(07):1715-20.

9. Thangarajah T, Prasad P S, Narayan B. Surgical site infections following open reduction and internal fixation of ankle fractures. Open Orthop J 2009;3(03):56-60.

10. Korim MT, Payne R, Bhatia MA. Case-control study of surgical site infection following operative fixation of fractures of the ankle in a large U.K. trauma unit. Bone Joint J 2014;96B:636-40.

11. Schepers T, De vries MR, Van Lieshout EM, Van der Elst M. The timing of ankle fracture surgery and the effect on infectious complications:A case series and systematic review of the literature. 2013 ;37(3): 489-494.

12. Miller AG, Margules A, Raikin SM, Risk factors for wound complications after ankle fracture surgery. J Bon Joint surg 2012; 21:94(22):2047-52. 
Open Reduction And Internal Fixation Of Ankle Fractures - Is Timing Of Surgery Important?

13. Adam G, Andrew M, Steven R. Risk Factors for wound complications after ankle fracture surgery. J Bon Joint Surg 2012;94(22):2047-52.

14. Saithna A, Moody W, Jenkinson E, Almazedi B, Sargeant I. The influence of timing of surgery on soft tissue complication in closed ankle fractures. Euro J Ortho Surg Traumatol 2009;19(7):481-484.

15. Weber BG. Die Verletzungen des oberen Sprunggelenkes (The injuries of the upper ankle), 2nd edition. Bern, Switzerland: Huber 1972, pp 34.

16. Todd B. New CDC guideline for the prevention of surgical site infection. Am J Nurs 2017;117:17.

17. Singh BI, Balaratnam S, Naidu V. Early versus delayed surgery for ankle fractures: A comparison of results. Eur J Orthop Surg Traumatol 2005;15:23-27.
18. Adamson SP, Trickett R, Hodgson P, Mohanty K. Ankle fractures: Impact of timing of surgery. Inj Extra 2009;40:224.

19. Pietzik P, Qureshi I, Langdon J, Molloy S, Solan M.Cost benefit with early operative fixation of unstable ankle fractures. 2006; 88(4): 405-407.

20. Singh RA, Trickett R, Hodgson P. Early versus late surgery for closed ankle fractures. J Orthop Surg 2015;23(3):341-4

21. Naumann MG, Sigurdsen U, Utvag SE, Stavem K. Associations of timing of surgery with postoperative length of stay, complications, and functional outcomes 3-6 years after operative fixation of closed ankle fractures. Injury 2017;48(7):1662-1669.

22. Sukeik M, Qaffaf M, Ferrier G. Ankle fractures: Impact of swelling on timing of surgery, length of hospital stay and the economic burden. Inj Extr 2010;41:133-137. 